PROCEEDINGS OF THE

AMERICAN MATHEMATICAL SOCIETY

Volume 126, Number 10, October 1998, Pages 3045-3053

S 0002-9939(98)04601-2

\title{
FIXED POINTS AND RANDOM FIXED POINTS FOR WEAKLY INWARD APPROXIMABLE MAPS
}

\author{
DONAL O'REGAN
}

(Communicated by Palle E. T. Jorgensen)

\begin{abstract}
In this paper we obtain new fixed point and random fixed point theory for approximable maps.
\end{abstract}

\section{INTRODUCTION}

This paper presents fixed point theory for weakly inward, approximable maps defined on a metrizable linear topological space (which is not necessarily locally convex). The paper is divided into two main parts. In Section 2 we use ideas in [1] (see also $[9,12])$ to present new fixed point results for approximable maps. These maps were introduced in the Hausdorff linear topological space setting in $[2,3]$. We will discuss in detail the setting when our map is also weakly inward; however other types of maps will also be considered. In Section 3 we use a recent result [14] to present random analogues of the results in Section 2.

We now gather together some definitions and known facts which will be used throughout this paper. Let $E$ be a Hausdorff linear topological vector space and let $C$ be a closed, convex subset of $E$. The set

$$
I_{C}(x)=\{x+\lambda(y-x): \lambda \geq 0, y \in C\} \quad \text { for } x \in C
$$

is called the inward set at $x$. A mapping $F: C \rightarrow 2^{E}$ (here $2^{E}$ denotes the family of all nonempty subsets of $E$ ) is said to be weakly inward with respect to $C$ if

$$
F(x) \cap \overline{I_{C}(x)} \neq \emptyset \text { on } C .
$$

In fact, since $C$ is closed and convex we have

$$
\overline{I_{C}(x)}=x+\overline{\{\lambda(y-x): \lambda \geq 1, y \in C\}} .
$$

Let $Y$ be a topological space. A mapping $F: E \rightarrow 2^{Y}$ is upper semicontinuous (u.s.c.) if the set $F^{-1}(B)=\{x \in E: F(x) \cap B \neq \emptyset\}$ is closed for any closed set $B$ in $Y$.

Suppose $X$ and $Z$ are subsets of Hausdorff topological vector spaces $E_{1}$ and $E_{2}$ respectively and $F: X \rightarrow 2^{Z}$ is a multifunction. Given two open neighborhoods $U$ and $V$ of the origins in $E_{1}$ and $E_{2}$ respectively, a $(U, V)$-approximative continuous selection [2] of $F$ is a continuous function $s: X \rightarrow Z$ satisfying

$$
s(x) \in(F[(x+U) \cap X]+V) \cap Z \text { for every } x \in X .
$$

Received by the editors December 26, 1996 and, in revised form, March 17, 1997.

1991 Mathematics Subject Classification. Primary 47H04, 47H10, 47H40, 54C60, 54H25.

(C)1998 American Mathematical Society 
$F$ is said to be approximable [3] if its restriction $\left.F\right|_{K}$ to any compact subset $K$ of $X$ admits a $(U, V)$-approximative continuous selection for every open neighborhoods $U$ and $V$ of the origins in $E_{1}$ and $E_{2}$ respectively.

Let $(\Omega, \mathcal{A})$ denote a measurable space. For a metric space $(X, d)$ we denote by $C(X)$ all nonempty closed subsets of $X$. Suppose $Z$ is a nonempty subset of $X$ and $F: Z \rightarrow 2^{X}$. Now $F$ is called hemicompact if each sequence $\left\{\left(x_{n}\right)\right\}_{n=1}^{\infty}$ in $Z$ has a convergent subsequence whenever $d\left(x_{n}, F\left(x_{n}\right)\right) \rightarrow 0$ as $n \rightarrow \infty$ (recall that if $Y$ is a nonempty subset of $X$ then $\left.d(x, Y)=\inf _{y \in Y} d(x, y)\right)$. A mapping $F: \Omega \rightarrow$ $2^{X}$ is measurable (respectively weakly measurable) if for every closed (respectively open) subset $D$ of $X, F^{-1}(D)=\{w \in \Omega: F(w) \cap D \neq \emptyset\} \in \mathcal{A}$. A mapping $F: \Omega \times Z \rightarrow 2^{X}$ is called a random operator if for every $x \in Z$, the map $F(., x)$ : $\Omega \rightarrow X$ is measurable. A random operator $F: \Omega \times Z \rightarrow 2^{X}$ is called continuous (hemicompact, etc.) if for each $w \in \Omega, F(w,$.$) is continuous (hemicompact, etc.).$ The single valued map $\phi: \Omega \rightarrow X$ is said to be (i) a deterministic fixed point of $F$ if $\phi(w) \in F(w, \phi(w))$ for all $w \in \Omega$ and (ii) a random fixed point of $F$ if $\phi$ is a measurable map such that $\phi(w) \in F(w, \phi(w))$ for all $w \in \Omega$.

Next we state a very recent result of Tan and Yuan [14].

Theorem 1.1. Let $(\Omega, \mathcal{A})$ be a measurable space and $Z$ a nonempty, separable, complete subset of a metric space $(X, d)$. Suppose the map $F: \Omega \times Z \rightarrow C(X)$ is a random, continuous, hemicompact map. Then $F$ has a deterministic fixed point iff $F$ has a random fixed point.

To conclude this introduction, we would like to state that the results of this paper will be used in forthcoming papers ("Fixed points, multivalued inequalities, control problems and differential inclusions on proximate retracts" and "Viable solutions of differential equations and inclusions on proximate retracts in Banach spaces") to establish new existence theory for viable solutions of differential inclusions on proximate retracts.

\section{FIXED POINTS}

This section presents some fixed point theory for upper semicontinuous maps. Throughout this section $E$ will be a metrizable (metric $d$ ) linear topological vector space (not necessarily locally convex), $C$ will be a closed, convex subset of $E$, and $U_{0}$ a $d$-bounded (i.e. there exists $K>0$ with $U_{0} \subset\{x \in E: d(0, x)<K\}$ ) open subset of $E$. We will let $U=U_{0} \cap C$ and $0 \in U$. We consider maps $F: \bar{U} \rightarrow$ $C C(E)$; here $C C(E)$ denotes the family of all nonempty, compact subsets of $E$. Our discussion is restricted to the spaces $\operatorname{IAP}_{C}(\bar{U}, E), K A P(\bar{U}, E), A P(\bar{U}, E)$ and $\operatorname{KIAP}(\bar{U}, E)$.

Definition 2.1. We say $F \in I A P_{C}(\bar{U}, E)$ if $F: \bar{U} \rightarrow C C(E)$ is a u.s.c., bounded

(i.e. $F(\bar{U})$ is a subset of a $d$-bounded set in $E$ ), approximable, weakly inward w.r.t. $C$, map which satisfies property (B).

Remark. Property (B) will usually mean that the map is compact. However in some situations (which we will specify at each stage) property (B) will mean that the map is condensing. We only introduce property (B) for convenience in presenting our results. 
Definition 2.2. We say $F \in K A P_{C}(\bar{U}, E)$ if $F: \bar{U} \rightarrow C K(E)$ is a u.s.c., bounded map which satisfies property (B) and

$$
F(x) \subseteq \overline{I_{C}(x)} \text { on } \bar{U}
$$

here $C K(E)$ denotes the family of all nonempty, compact, acyclic $[6,7]$ subsets of E.

Definition 2.3. We say $F \in A P_{C}(\bar{U}, E)$ if $F: \bar{U} \rightarrow C C(E)$ is a u.s.c., bounded, approximable map which satisfies property $(\mathrm{B})$ and

$$
F(x) \subseteq \overline{I_{C}(x)} \text { on } \bar{U} \text {. }
$$

Definition 2.4. We say $F \in K I A P_{C}(\bar{U}, E)$ if $F: \bar{U} \rightarrow C K(E)$ is a u.s.c., bounded, weakly inward w.r.t. $C$, map which satisfies property (B).

Theorem 2.1. Let $E, C, U_{0}, U$ be as above, $0 \in U$ and $G \in I A P_{C}(\bar{U}, E)$. Assume the following conditions are satisfied:

$$
\text { if } \begin{aligned}
A \subseteq \bar{U} \text { satisfies } A \subseteq \operatorname{co}(G(A) \cup\{0\}) \text {, then } \bar{A} \text { is compact, } \\
\left\{\begin{array}{l}
\text { any map } F \in I A P_{\Omega}(\Omega, E) \text { has a fixed point; here } \\
\Omega \text { is any } d \text {-bounded, closed, convex subset of } E,
\end{array}\right.
\end{aligned}
$$

and

$$
\left\{\begin{array}{l}
\text { for any continuous map } \mu: \bar{U} \rightarrow[0,1] \text { with } \mu(\partial U)=0 \\
\text { and any d-bounded, closed, convex set } \Omega \supseteq \bar{U} \\
\text { we have that } N: \Omega \rightarrow C C(E) \text { (given below) } \\
\text { satisfies property }(B) ;
\end{array}\right.
$$

here $\partial U$ denotes the boundary of $U$ in $C$ and

$$
N(x)=\left\{\begin{array}{l}
\mu(x) G(x), x \in \bar{U} \\
\{0\}, \quad x \in \Omega \backslash \bar{U}
\end{array}\right.
$$

Then either

(A1) $G$ has a fixed point in $\bar{U}$; or

(A2) there exist $u \in \partial U$ and $\lambda \in(0,1)$ with $u \in \lambda G(u)$.

Remark. Suppose $E$ is a Banach space, property (B) means the map is condensing and $G: \bar{U} \rightarrow C Q(E)$; here $C Q(E)$ denotes the family of all nonempty, compact, convex subsets of $E$. That is, $G \in \operatorname{IAP}_{C}(\bar{U}, E)$ if $G: \bar{U} \rightarrow C Q(E)$ is a u.s.c., bounded, condensing, weakly inward w.r.t. $C$, map (it is well known $[3,4]$ that $G$ is approximable). In addition (2.1), (2.2) (see [5]) and (2.3) are clearly satisfied. We refer the reader also to [13].

Proof. Suppose (A2) does not hold and $G$ does not have a fixed point on $\partial U$. Let

$$
H=\{x \in \bar{U}: x \in \lambda G(x) \text { for some } \lambda \in[0,1]\} \text {. }
$$

Now $H \neq \emptyset$ since $0 \in H$. A standard argument [10] implies $H$ is closed. This together with (2.1) implies $H$ is compact. Now since $H \cap \partial U=\emptyset$ there is a continuous function $\mu: \bar{U} \rightarrow[0,1]$ with $\mu(H)=1$ and $\mu(\partial U)=0$. Since $U_{0}$ is a $d$-bounded set and $G \in I A P_{C}(\bar{U}, E)$ (in particular $G(\bar{U})$ is a subset of a $d$-bounded set in $E$ ), there exists $R>0$ with

$$
U_{0} \subset\{x \in E: d(0, x)<R\} \quad \text { and } \quad G(\bar{U}) \subset\{x \in E: d(0, x)<R\} .
$$


Let $\Omega=C \cap\{x \in E: d(0, x)<R+1\}$ and define

$$
N(x)=\left\{\begin{array}{l}
\mu(x) G(x), x \in \bar{U} \\
\{0\}, \quad x \in \bar{\Omega} \backslash \bar{U}
\end{array}\right.
$$

Now $N: \bar{\Omega} \rightarrow C C(E)$ is u.s.c., bounded, and satisfies property (B). Next we show that $N$ is approximable. To see this, let $K$ be any compact subset of $\bar{\Omega}$. Our argument follows the technique in [2, page 486] (see also [12]). Let $U_{1}$ and $V_{1}$ be two open neighborhoods of the origin. We may assume without loss of generality that $U_{1}$ is symmetric. Let $V_{2} \subseteq V_{1}$ be a balanced open neighborhood of the origin such that $V_{2}+V_{2} \subseteq V_{1}$. Since $K$ is compact, $G$ is u.s.c., and $\mu$ is continuous, then for any $x \in K$, it is easy to see (since $G(K)$ is a subset of a bounded set) that there exists a neighborhood $W_{x} \subseteq U_{1}$ of the origin such that

$$
\left.\left.\mu\left(\left[x+W_{x}\right)\right] \cap K\right) G\left(\left[x+W_{x}\right)\right] \cap K\right) \subseteq \mu(x) G(x)+V_{2} ;
$$

here we use the notation $\mu(A) G(A)=\{\mu(x) G(y): x \in A, y \in A\}$. Let $Z_{x} \subseteq W_{x}$ be a neighborhood of the origin such that $Z_{x}+Z_{x} \subseteq W_{x}$. Now let $\left\{x_{i}+Z_{x_{i}}\right\}_{1}^{n}$ be an open cover of $K$ and let $U_{2}=\bigcap_{1}^{n} Z_{x_{i}}$. Since $K \cap \bar{U}$ is compact, let $s: K \cap \bar{U} \rightarrow E$ be the $\left(U_{2}, V_{2}\right)$-approximative continuous selection of $\left.G\right|_{K \cap \bar{U}}$. Let

$$
s_{1}(x)=\left\{\begin{array}{l}
\mu(x) s(x), x \in K \cap \bar{U}, \\
0, \quad x \in K \cap[\bar{\Omega} \backslash \bar{U}] .
\end{array}\right.
$$

We now check that $s_{1}: K \rightarrow E$ is a $\left(U_{1}, V_{1}\right)$-approximative continuous selection of $\left.N\right|_{K}$. Fix $x \in K \cap \bar{U}$. Then $x \in x_{i}+Z_{x_{i}}$ for some $i \in\{1, \ldots, n\}$. Now since

$$
s(x) \in G\left(x+U_{2}\right)+V_{2}
$$

we have

$$
\mu(x) s(x) \in \mu\left(x_{i}+W_{x_{i}}\right) G\left(x+U_{2}\right)+V_{2},
$$

and so

$$
\mu(x) s(x) \in \mu\left(x_{i}+W_{x_{i}}\right) G\left(x_{i}+W_{x_{i}}\right)+V_{2} .
$$

Thus

$$
\mu(x) s(x) \in \mu G\left(x_{i}\right)+V_{1},
$$

and so

$$
\mu(x) s(x) \in \mu G\left(\left[x+U_{1}\right] \cap K\right)+V_{1} .
$$

Consequently $s_{1}: K \rightarrow E$ is a $\left(U_{1}, V_{1}\right)$-approximative continuous selection of $\left.N\right|_{K}$. We claim that $N$ is weakly inward w.r.t. $\bar{\Omega}$. If the claim is true then $N \in I A P_{\bar{\Omega}}(\bar{\Omega}, E)$, and so by (2.2) we know that there exists $x \in \bar{\Omega}$ with $x \in N(x)$. However, since $0 \in U$ we have $x \in U$. Thus $x \in N(x)$ and $x \in U$. Consequently

$$
x \in \lambda G(x) \text { with } 0 \leq \lambda=\mu(x) \leq 1 .
$$

As a result $x \in H$, so $\mu(x)=1$. Thus $x \in G(x)$, and we are finished.

It remains to prove the claim. If $x \in \bar{\Omega} \backslash \bar{U}$ then $N(x)=\{0\} \in \overline{I_{\bar{\Omega}}(x)}$, since $0 \in U_{0} \cap C($ so $0 \in \Omega)$. Next fix $x \in \bar{U}$. Now since

$$
G(x) \cap \overline{I_{C}(x)} \neq \emptyset \text { and } 0 \in \overline{I_{C}(x)},
$$

then

$$
\left.[s G(x)+(1-s) 0] \cap \overline{I_{C}(x)} \neq \emptyset \quad \text { (where } s=\mu(x)\right)
$$

since $\overline{I_{C}(x)}$ is convex. Consequently

$$
N(x) \cap \overline{I_{C}(x)} \neq \emptyset .
$$


Now take $y \in N(x)=\mu(x) G(x)$ and $y \in \overline{I_{C}(x)}$. Then there exist $\left\{\lambda_{n}\right\}_{n \in \mathbf{N}}$, with $\lambda_{n} \geq 1$ for $n \in \mathbf{N}$, and $\left\{z_{\lambda_{n}}\right\}_{n \in \mathbf{N}} \subseteq C$, with

$$
d\left(y, x+\lambda_{n}\left[z_{\lambda_{n}}-x\right]\right) \rightarrow 0 \quad \text { as } n \rightarrow \infty .
$$

Let

$$
v_{\lambda_{n}}=x+\lambda_{n}\left[z_{\lambda_{n}}-x\right]
$$

Now $v_{\lambda_{n}} \rightarrow y$ as $n \rightarrow \infty$, so

$$
v_{\lambda_{n}} \in\{x \in E: d(0, x)<R+1\} \text { for } n \in \mathbf{N} \text { sufficiently large. }
$$

Let $\tau_{n}=\frac{1}{\lambda_{n}}$. Then

$$
z_{\lambda_{n}}=\left(1-\tau_{n}\right) x+\tau_{n} v_{\lambda_{n}}
$$

so $z_{\lambda_{n}} \in \bar{\Omega}$ for $n$ sufficiently large. Thus

$$
z_{\lambda_{n}} \in \bar{\Omega} \text { for } n \text { sufficiently large and } d\left(y, x+\lambda_{n}\left[z_{\lambda_{n}}-x\right]\right) \rightarrow 0 \text { as } n \rightarrow \infty \text {. }
$$

Thus $y \in \overline{I_{\bar{\Omega}}(x)}$.

Remark. It is possible to replace $U_{0} d$-bounded and $G(\bar{U})$ a subset of a $d$-bounded set in $E$ by: there exists a convex set $C_{0}$ with $U_{0} \subset C_{0}$ and $G(\bar{U}) \subset C_{0}$ [of course the other assumptions have to be appropriately adjusted]. A similar remark applies to the other theorems in this paper.

Essentially the same reasoning as in Theorem 2.1 (except easier) establishes the corresponding results for $K A P$ maps.

Theorem 2.2. Let $E, C, U_{0}, U$ be as above, $0 \in U$ and $G \in K_{A} P_{C}(\bar{U}, E)$. Suppose (2.1) holds. In addition assume that the conditions

$$
\left\{\begin{array}{l}
\text { any map } F \in K A P_{\Omega}(\Omega, E) \text { has a fixed point; } \\
\text { here } \Omega \text { is any } d \text {-bounded, closed, } \\
\text { convex subset of } E
\end{array}\right.
$$

and

$$
\left\{\begin{array}{l}
\text { for any continuous map } \mu: \bar{U} \rightarrow[0,1] \text { with } \mu(\partial U)=0 \\
\text { and any d-bounded, closed, convex set } \Omega \supseteq \bar{U} \\
\text { we have that } N: \Omega \rightarrow C K(E) \text { (given below) } \\
\text { satisfies property }(B)
\end{array}\right.
$$

are satisfied; here

$$
N(x)=\left\{\begin{array}{l}
\mu(x) G(x), x \in \bar{U} \\
\{0\}, \quad x \in \Omega \backslash \bar{U}
\end{array}\right.
$$

Then either

(A1) $G$ has a fixed point in $\bar{U}$; or

(A2) there exist $u \in \partial U$ and $\lambda \in(0,1)$ with $u \in \lambda G(u)$.

Remark. Suppose $E$ is a uniformly convex Banach space (or more generally a reflexive Banach space satisfying property $(\mathrm{H})$ in $[6])$, and property $(\mathrm{B})$ means the map is compact. That is, $G \in K A P_{C}(\bar{U}, E)$ if $G: \bar{U} \rightarrow C K(E)$ is a u.s.c., compact map with $F(x) \subseteq \overline{I_{C}(x)}$ on $\bar{U}$. Now (2.1), (2.4) (see [6, page 22]) and (2.5) are clearly satisfied. If $E$ is a Hilbert space we could replace $G$ being compact with $G$ being condensing (see $[6]$ ). We refer the reader also to $[8,9]$.

Similarly we have results for $A P$ and $K I A P$ maps. 
Theorem 2.3. Let $E, C, U_{0}, U$ be as above, $0 \in U$ and $G \in A P_{C}(\bar{U}, E)$. Suppose (2.1) and (2.3) hold. In addition assume that the condition

$$
\left\{\begin{array}{l}
\text { any map } F \in A P_{\Omega}(\Omega, E) \text { has a fixed point; here } \\
\Omega \text { is any d-bounded, closed, convex subset of } E
\end{array}\right.
$$

is satisfied. Then either

(A1) $G$ has a fixed point in $\bar{U}$; or

(A2) there exist $u \in \partial U$ and $\lambda \in(0,1)$ with $u \in \lambda G(u)$.

Theorem 2.4. Let $E, C, U_{0}, U$ be as above, $0 \in U$ and $G \in \operatorname{KIAP}_{C}(\bar{U}, E)$. Suppose (2.1) and (2.5) hold. In addition assume that the condition

$$
\left\{\begin{array}{l}
\text { any map } F \in K I A P_{\Omega}(\Omega, E) \text { has a } \\
\text { fixed point; here } \Omega \text { is any d-bounded, } \\
\text { closed, convex subset of } E
\end{array}\right.
$$

is satisfied. Then either

(A1) $G$ has a fixed point in $\bar{U}$; or

(A2) there exist $u \in \partial U$ and $\lambda \in(0,1)$ with $u \in \lambda G(u)$.

If $E$ is Fréchet and $G$ is a condensing map, then (2.1) is automatically satisfied. However (2.1) may not be satisfied if $G$ is a 1-set contractive map. It is of interest to try to extend Theorems 2.1-2.4 to this setting. We will just state and prove a result for $I A P$ maps. A similar result holds for $K A P, A P$ and $K I A P$ maps (we choose to omit the details, since the reasoning is essentially the same in all cases).

Theorem 2.5. Let $E, C, U_{0}, U$ be as above, $0 \in U$ and $G: \bar{U} \rightarrow C C(E)$ be a u.s.c., bounded, approximable, weakly inward w.r.t. C, map. Assume the following conditions are satisfied:

$$
\begin{aligned}
\text { for each } & n \in\{2,3, \ldots\}, G_{n}=\left(1-\frac{1}{n}\right) G \text { satisfies property }(B) ; \\
& \left\{\begin{array}{l}
\text { any map } F \in I A P_{\Omega}(\Omega, E) \text { has a fixed point; } \\
\text { here } \Omega \text { is any } d-\text { bounded, closed, } \\
\text { convex subset of } E ;
\end{array}\right. \\
& \left\{\begin{array}{l}
\text { for each fixed } n \in\{2,3, \ldots\}, \text { if } A \subseteq \bar{U} \text { satisfies } \\
A \subseteq \text { co }\left(G_{n}(A) \cup\{0\}\right), \text { then } \bar{A} \text { is compact }
\end{array}\right. \\
& \left\{\begin{array}{l}
\text { for each fixed } n \in\{2,3, \ldots\}, \text { and for any } \\
\text { continuous map } \mu: \bar{U} \rightarrow[0,1] \text { with } \mu(\partial U)=0 \\
\text { and any d-bounded, closed, convex set } \\
\Omega \supseteq \bar{U}, \text { we have that } N_{n}: \Omega \rightarrow C C(E) \\
(\text { given below) satisfies property }(B)
\end{array}\right.
\end{aligned}
$$

and

$$
\left\{\begin{array}{l}
\text { if }\left\{x_{n}\right\} \subseteq \bar{U} \text { with } y_{n} \in G\left(x_{n}\right) \text { for all } n \\
\text { and } x_{n}-y_{n} \rightarrow 0 \text { as } n \rightarrow \infty, \\
\text { then there exists } x \in \bar{U} \text { with } x \in G(x) ;
\end{array}\right.
$$


here

$$
N_{n}(x)=\left\{\begin{array}{l}
\mu(x) G_{n}(x), x \in \bar{U} \\
\{0\}, \quad x \in \Omega \backslash \bar{U}
\end{array}\right.
$$

Then either

(A1) $G$ has a fixed point in $\bar{U}$; or

(A2) there exist $u \in \partial U$ and $\lambda \in(0,1)$ with $u \in \lambda G(u)$.

Proof. Suppose (A2) does not hold. For each $n \in\{2,3, \ldots\}$ let $G_{n}=\left(1-\frac{1}{n}\right) G$. We claim $G_{n} \in I A P_{C}(\bar{U}, E)$ for each $n \in\{2,3, \ldots\}$. First we show that $G_{n}$ is weakly inward w.r.t. $C$. To see this, fix $x \in \bar{U}$. Now since

$$
G(x) \cap \overline{I_{C}(x)} \neq \emptyset, \quad 0 \in \overline{I_{C}(x)}
$$

and $G_{n}(x)=\left(1-\frac{1}{n}\right) G(x)+\frac{1}{n} 0$, we have

$$
G_{n}(x) \cap \overline{I_{C}(x)} \neq \emptyset
$$

since $\overline{I_{C}(x)}$ is convex. Consequently $G_{n}$ is weakly inward w.r.t. $C$. It remains to show that $G_{n}$ is approximable. To see this let $K$ be any compact subset of $\bar{U}$. Let $U_{1}$ and $V_{1}$ be two open neighborhoods of the origin. We may assume without loss of generality that $V_{1}$ is balanced. Let $s: K \rightarrow E$ be the $\left(U_{1}, V_{1}\right)$-approximative continuous selection of $\left.G\right|_{K}$, i.e.

$$
s(x) \in G\left(x+U_{1}\right)+V_{1} .
$$

Now clearly $\left(1-\frac{1}{n}\right) s: K \rightarrow E$ is a $\left(U_{1}, V_{1}\right)$-approximative continuous selection of $\left.\left(1-\frac{1}{n}\right) G\right|_{K}$.

Consequently $G_{n} \in I A P_{C}(\bar{U}, E)$. We would like to apply Theorem 2.1. If there exist $u \in \partial U$ and $\lambda \in(0,1)$ with $u \in \lambda G_{n}(u)$, then $u \in \lambda\left(1-\frac{1}{n}\right) G(u)$. This is a contradiction since (A2) does not hold. Apply Theorem 2.1 (with $G_{n}$ replacing $G)$, and we are guaranteed that $G_{n}$ has a fixed point $x_{n} \in \bar{U}$. Choose $y_{n} \in G\left(x_{n}\right)$ with $x_{n}=\left(1-\frac{1}{n}\right) y_{n}$. Notice that $x_{n}-y_{n}=-\frac{1}{n} y_{n} \rightarrow 0$, since $G(\bar{U})$ is bounded. Now apply (2.12) to deduce that there exists $x \in \bar{U}$ with $x \in G(x)$.

\section{RANDOM FIXED POINTS}

We now use Theorem 1.1 to produce random analogues of the results in Section 2. We prove a result for $I A P$ maps (i.e., we will present the random analogue of Theorem 2.1). Similarly, random fixed point theorems coould be presented for $K A P, A P$ and KIAP maps (i.e. we could establish the random analogue of Theorems 2.2$2.5)$; however we choose to omit the details since the reasoning is essentially the same in all cases.

Throughout $E$ will be a metrizable (metric $d$ ) linear topological vector space (not necessarily locally convex), $C$ will be a closed, convex subset of $E$, and $U_{0}$ a $d$-bounded open subset of $E$. We will let $U=U_{0} \cap C$ and $0 \in U$.

Theorem 3.1. Let $E, C, U_{0}, U$ be as above and $0 \in U$. Also $(\Omega, \mathcal{A})$ is a measurable space and $\bar{U}$ is a separable, complete subset of $E$. Assume $G: \Omega \times \bar{U} \rightarrow$ $C C(E)$ is a continuous, bounded, approximable, weakly inward w.r.t. $C$, hemicompact map which satsifies property $(B)$ (this means that, for each $w \in \Omega, G(w,$. ) 
is a continuous, bounded, approximable, weakly inward w.r.t. $C$, hemicompact map which satsifies property (B)). Suppose the following conditions are satisfied:

$$
\begin{gathered}
\left\{\begin{array}{l}
\text { for each } w \in \Omega, \text { if } A \subseteq \bar{U} \text { satisfies } \\
A \subseteq \operatorname{co}(G(w, A) \cup\{0\}) \text { then } \bar{A} \text { is compact; }
\end{array}\right. \\
\left\{\begin{array}{l}
\text { for each } w \in \Omega, \text { any map } F(w, .) \in I A P_{\Omega_{0}}\left(\Omega_{0}, E\right) \\
\text { has a fixed point; here } \Omega_{0} \text { is any d-bounded, } \\
\text { closed, convex subset of } E ;
\end{array}\right. \\
\left\{\begin{array}{l}
\text { for each } w \in \Omega, \text { and for any continuous map } \\
\mu: \bar{U} \rightarrow[0,1] \text { with } \mu(\partial U)=0 \text { and } \\
\text { any } d-\text {-bounded, closed, convex set } \Omega_{0} \supseteq \bar{U}, \\
\text { we have that } N(w, .): \Omega_{0} \rightarrow C C(E) \\
\text { (given below) satisfies property }(B) ;
\end{array}\right.
\end{gathered}
$$

and

$$
\text { for any } w \in \Omega, u \notin \lambda G(w, u) \text { for all } u \in \partial U \text { and } \lambda \in(0,1) \text {; }
$$

here

$$
N(w, x)=\left\{\begin{array}{l}
\mu(x) G(w, x), x \in \bar{U} \\
\{0\}, x \in \Omega_{0} \backslash \bar{U}
\end{array}\right.
$$

Then $G$ has a random fixed point.

Proof. From Theorem 2.1, we know that $G$ has a deterministic fixed point. Now Theorem 1.1 implies $G$ has a random fixed point.

\section{REFERENCES}

1. J. Banas and K. Goebel, Measures of noncompactness in Banach spaces, Marcel Dekker, New York, 1980. MR 82f:47066

2. H. Ben-El-Mechaiekh and P.Deguire, Approachability and fixed points for non-convex setvalued maps, Jour. Math. Anal. Appl., 170(1992), 477-500. MR 94a:54103

3. H. Ben-El-Mechaiekh and A.Idzik, A Leray-Schauder type theorem for approximable maps, Proc. Amer. Math. Soc., 122(1994), 105-109. MR 94k:54074

4. A. Cellina, A theorem on the approximation of compact multivalued mappings, Atti. Accad. Naz. Lincei Rend., 47(1969), 429-433. MR 43:2676

5. K. Deimling, Multivalued differential equations, Walter de Gruyter, Berlin, 1992. MR 94b:34026

6. P. M. Fitzpatrick and W. V. Petryshyn, Fixed point theorems for multivalued noncompact acyclic mappings, Pacific Jour. Math., 54(1974), 17-23. MR 53:8973

7. L. Gorniewicz, A. Granas and W. Kryszewski, Sur la méthode de l'homotopie dans la théorie des points fixes pour les applications multivoques (partie 1: Transversalité topologique), $C$. R. Acad. Sci. Paris, Ser. 1, 307(1988), 489-492. MR 90g:55002

8. M. Lassonde, On the use of KKM multifunctions in fixed point theory and related topics, Jour. Math. Anal. Appl., 97(1983), 151-201. MR 84k:47049

9. D. O'Regan, Some fixed point theorems for concentrative mappings between locally convex linear topological spaces, Nonlinear Analysis, 27(1996), 1437-1446. MR 97i:47121

10. D. O'Regan, A topological approach to integral inclusions, Proc. Royal Irish Acad., 97A (1997), 101-111.

11. D. O'Regan, A continuation theory for weakly inward mappings, Glasgow Math. Journal, to appear.

12. S. Park, Fixed point for approximable maps, Proc. Amer. Math. Soc., 124(1996), 3109-3114. MR 96m: 47108 
13. S. Reich, A fixed point theorem in Fréchet spaces, Jour. Math. Anal. Appl., 78(1980), 33-35. MR 82h: 47055

14. K. K. Tan and X. Z. Yuan, Random fixed point theorems and approximation in cones, Jour. Math. Anal. Appl., 185(1994), 378-390. MR 95d:47085

Department of Mathematics, University College Galway, Galway, Ireland

E-mail address: donal.oregan@ucg.ie 